EFI-95-16

hep-th/9504047

\title{
THE HETEROTIC STRING IS A SOLITON
}

\author{
Jeffrey A. Harvey \\ Enrico Fermi Institute, University of Chicago \\ 5640 Ellis Avenue, Chicago, IL 60637 \\ Internet: harvey@poincare.uchicago.edu \\ Andrew Strominger \\ Department of Physics \\ University of California \\ Santa Barbara, CA 93206-9530 \\ Internet: andy@denali.physics.ucsb.edu
}

\begin{abstract}
It is shown that the Type IIA superstring compactified on $K 3$ has a smooth string soliton with the same zero mode structure as the heterotic string compactified on a four torus, thus providing new evidence for a conjectured exact duality between the two sixdimensional string theories. The chiral worldsheet bosons arise as zero modes of RamondRamond fields of the IIA string theory and live on a signature $(20,4)$ even, self-dual lattice. Stable, finite loops of soliton string provide the charged Ramond-Ramond states necessary for enhanced gauge symmetries at degeneration points of the $K 3$ surface. It is also shown that Type IIB strings toroidally compactified to six dimensions have a multiplet of string solutions with Type II worldsheets.
\end{abstract}

April, 1995 


\section{Introduction}

The idea that fundamental strings might be viewed as solitons is an old one dating back at least to the work of Nielsen and Olesen [1]. While past attempts to describe fundamental strings as solitons in an ordinary field theory have not been fruitful, from the current perspective one might hope to find them as solitons in another dual string theory. Some weak evidence for such a possibility was presented in [2, 3] where certain analogies between fundamental strings and solitons were drawn.

However duality in the geometrical sense does not usually relate a fundamental string to a soliton string. A simple generalization [4] of Dirac's argument shows that, in $d$ spacetime dimensions, a $p$-brane 1 is dual to a $(d-p-4)$-brane (Thus in ten dimensions a fivebrane is dual to a string and should couple to the dual form of supergravity [5].). Unobservability of the Dirac $(d-p-3)$-brane emanating from the $p$-brane (i.e. the generalized Dirac string) implies that the minimal charge carried by the dual $(d-p-4)$ brane is inversely proportional to the charge carried by the $p$-brane. Thus the $(d-p-$ 4)-brane will be weakly coupled when the $p$-brane is strongly coupled and vice versa. Motivated in part by this observation it was conjectured in [6] that fivebrane solitons should be relevant for the description of the strongly coupled phase of fundamental tendimensional string (onebrane) theory. This is the natural generalization to ten-dimensional string theory of the Montonen-Olive conjecture for $d=4$ Yang-Mills theory. However as very little is understood about the quantum theory of fivebranes (see [7] for a review) this conjecture is difficult to test.

In six dimensions, a string is dual to a string and the prospects for a duality which can be understood concretely are much more promising [8,9]. In addition, as discussed in [10,8,9 2, string-string duality in six dimensions can be used to deduce $S$-duality in four-dimensional toroidally compactified heterotic string theory, because it transforms $S$ duality into the perturbatively manifest $T$-duality of the dual theory. This is referred to as the "duality of dualities". Evidence for $S$-duality of the toroidally compactified heterotic string has been accumulating [11] and there have been a variety of tantalizing hints of connections between this $S$-duality and dualities in various dimensions involving

1 We adopt the standard terminology that a $p$-brane is an extended object with $p$ spatial dimensions.

2 Reference [10] derived these results using a toroidally compactified version of string-fivebrane duality which is equivalent to string-string duality in six dimensions. 
strings and/or higher $p$-branes [12,13]. In [9] a coherent picture was presented of how these various dualities should be related to each other. One important lesson is that a consistent picture of strong coupling string dynamics in dimensions four through seven emerges as a consequence of a six-dimensional duality between the Type IIA string on $K 3$ and the heterotic string on $T^{4}$.

In this paper we shall investigate the worldsheet action for string solitons arising in several six-dimensional string theories. These string solitons are simply the fivebrane solitons of [6, 14, 15, 16] with four of their dimensions wrapped around the internal fourspace used in the compactification from ten to four dimensions, and correspond to exact conformal field theories [15].

We first consider the nonchiral IIA theory on K3. Assuming a quantization condition on Ramond-Ramond charge, we will show that the world sheet theory of the string soliton has a chiral structure with right-moving supersymmetry. In addition to the four transverse bosonic zero modes and eight right-moving fermion zero modes there are 20 leftmoving and 4 right-moving bosonic zero modes which lie on an even, self-dual Lorentzian lattice with signature $(20,4)$. This is precisely the structure of the heterotic string with a generic $T^{4}$ compactification! The soliton string couples to the dual Kalb-Ramond field and has string tension proportional to $e^{-2 \phi} / \alpha^{\prime}$ so that the fundamental string loop coupling constant becomes the dual worldsheet sigma-model expansion parameter [6, 17, 16]. We will also identify closed string solitons which become massless and lead to enhanced gauge symmetries at special points in the $K 3$ moduli space, in accord with conjectures in [13, 18,9].

The emergence of the heterotic string as a soliton is quite remarkable. While the existence of a rank-three field strength more or less implies the existence of string solitons in six dimensions, there is no guarantee that the effective worldsheet dynamics describe a critical string theory, let alone the intricate chiral structure of the heterotic string. It is especially striking that such a structure arises from the non-chiral IIA string.

Upon further toroidal compactification to four dimensions, the "duality of dualities" implies that the $T$-duals of the BPS states [2] of our type II string soliton are the $S$-duals of the BPS states of the toroidally compactified heterotic string. Thus in principle our results provide the solution to the $H$-monopole problem, but it remains to be understood how this works in detail. We hope to report on this elsewhere.

We will also show that the worldsheet action for the chiral type IIB string compactified on $T^{4}$ is a type II string with a $T^{4}$ target space as are the string solitons of the IIA theory. 
These results provide evidence in favor of (and were in part motivated by) the recent conjectures that the IIB theory is dual to itself while the IIA theory on $K 3$ is dual to the heterotic string on $T^{4}$ [13,9, 19]. The IIA-heterotic duality conjectures were based largely on the the earlier observation [20] that equivalence of the low-energy effective theories follows from symmetry considerations and on the consistent picture of dynamics in other dimensions that it implies. The emergence of the heterotic string as a soliton requires several "miracles" that do not follow from symmetry considerations alone and thus provides substantial new evidence for exact string-string duality. We will also find similar evidence for the conjecture [13,21,9] that eleven-dimensional supergravity is dual to the heterotic string compactified on $T^{3}$.

While these results are intriguing, a number of puzzles remain. One of these concerns toroidally compactified heterotic strings. The conjectured six-dimensional IIA - heterotic duality suggests that the heterotic string should have a string soliton with a worldsheet which is that of a IIA theory with a $K 3$ target. This structure does not appear to emerge in any simple way. Indeed, as pointed out in [8], toroidal compactification of the heterotic fivebrane seems to lead to a non-critical string 3 . This puzzle is related to the $H$-monopole problem [23] encountered in attempts to verify four-dimensional $S$-duality, since the $H$ monopoles arise in compactification of the six-dimensional string solitons. It seems likely that this puzzle can be addressed by a generalization of the techniques discussed here, at least at special points in the $K 3$ moduli space.

A second puzzle is the relation of this string-string duality to the string-fivebrane duality of [6]. The fact that the string solitons are compactified fivebranes clearly suggests a connection: string-string duality may be viewed as a compactified, six-dimensional version of string-fivebrane duality. However while in six dimensions it is sensible to describe the strongly coupled dynamics by a perturbative quantization which treats the weakly-coupled string solitons as fundamental objects, it is not clear if an analogous description is sensible in ten dimensions since we do not know how to quantize fundamental fivebranes. The proper role of fivebranes in nonperturbative string dynamics thus remains a mystery.

Finally, it is worth pointing out that the proposed IIA- heterotic string duality takes the smooth soliton solution described herein into a singular solution which describes the field around a fundamental heterotic string [3]. It has been appreciated for some time that the question of the singularity of the fundamental string solution depends on the

3 Presumably of the general variety discussed in [22]. 
metric used and that in the dual, or "fivebrane" metric the solution is in fact non-singular [16, 12, in harmony with the idea that fundamental strings are solitons of a dual theory. It has also been appreciated for some time [24], that properties of the low-energy effective theory imply that the low-energy fields outside a heterotic string could carry long range fields corresponding to the possibility of charge excitations on the string. The key missing ingredient in these previous observations was identification of the source of these long range fields. In the present work we fill this gap by showing that the full chiral zero mode structure of the fundamental heterotic string arises directly in the dual theory through well defined, normalizable zero modes of a non-singular soliton solution. With this in hand one can quantize the solitons as elementary objects and verify that their spectrum is precisely that required for an exact string-string duality.

The organization of this paper is as follows. Section 2 contains the construction of string solitons in the Type IIA theory on $T^{4}$ and $K 3$. We show that the heterotic string arises as a soliton in the $K 3$ case and relate the zero mode structure to the intersection matrix of $K 3$ and quantization of Ramond-Ramond (RR) charge. We also argue that the quantum of RR charge must have a specific value. Section 3 summarizes an analogous construction in eleven-dimensional supergravity compactified on $K 3$. Section 4 extends these results to the Type IIB theory on $T^{4}$ by constructing string solitons with Type II worldsheets and in Section 5 we mention an extension of these solutions which follows from an $S L(5, Z)$ duality of the Type IIB theory on $T^{4}$. In Section 6 we summarize our results and discuss some of their implications. We point out that finite loops of soliton string carry $\mathrm{RR}$ charge and hence must be stable and at special points in the $K 3$ moduli space can provide the massless states with the required degeneracies and quantum numbers needed for enhanced gauge symmetry.

\section{Heterotic String as a Soliton in Type IIA String Theory}

In ten dimensions the IIA string theory [25] contains a two-form field strength $G$ and a four-form field strength $F$ as well as the three-form $H$. The bosonic part of the low-energy 
action is (in sigma-model variables)

$$
\begin{aligned}
& S=\frac{1}{16 \pi \alpha^{\prime 4}} \int d^{10} x \sqrt{-g}\left[e^{-2 \phi}\left(R+4(\nabla \phi)^{2}-\frac{1}{3} H^{2}\right)-\alpha^{\prime} G^{2}-\frac{\alpha^{\prime}}{12} F^{\prime 2}\right. \\
& \left.-\frac{\alpha^{\prime}}{288} \epsilon^{M_{1} \ldots M_{10}} F_{M_{1} M_{2} M_{3} M_{4}} F_{M_{5} M_{6} M_{7} M_{8}} B_{M_{9} M_{10}}\right] \\
& =\frac{1}{16 \pi \alpha^{\prime 4}} \int d^{10} x \sqrt{-g} e^{-2 \phi}\left(R+4(\nabla \phi)^{2}\right) \\
& -\frac{1}{8 \pi \alpha^{\prime 4}} \int\left(e^{-2 \phi} H \wedge * H+\alpha^{\prime} G \wedge * G+\alpha^{\prime} F^{\prime} \wedge * F^{\prime}+2 \alpha^{\prime} F \wedge F \wedge B\right) .
\end{aligned}
$$

where $G=d A, \quad H=d B, F=d C$ and $F^{\prime}=d C+2 A \wedge H$.

The spacetime of the string soliton has the form

$$
K \times R^{4} \times M^{1,1}
$$

where $R^{4}$ is the space transverse to the string and $M^{1,1}$ is the string world-sheet. The string vacuum equations of motion require that $K$ be a Ricci-flat hyperkahler manifold, i.e. $R^{4}$, the four-torus $T^{4}$ or a $K 3$ surface. We will consider the latter two possibilities $\left(R^{4}\right.$ leads back to fivebranes). When necessary we will denote the coordinates on the spacetime (2.2) by $X^{M}=\left(y^{a}, x^{\mu}, \sigma^{\alpha}\right)$. The string soliton solution is given by choosing a Ricci-flat hyperkahler metric on $K$, a flat metric on $M^{1,1}$, and the following field configuration on the transverse space:

$$
\begin{aligned}
e^{2 \phi} & =e^{2 \phi_{0}}+\frac{\alpha^{\prime}}{x^{2}}, \\
H_{\mu \nu \lambda} & =-\epsilon_{\mu \nu \lambda}{ }^{\rho} \nabla_{\rho} \phi \\
g_{\mu \nu} & =e^{2 \phi} \delta_{\mu \nu} .
\end{aligned}
$$

with all other fields vanishing. This soliton string carries the minimal "magnetic" charge

$$
Q=-\frac{1}{2 \pi^{2}} \int_{S^{3}} H=\alpha^{\prime}
$$

in contrast to the "electric" charge carried by the fundamental Type IIA string. Note that the solution is presented in coordinates in which the metric is asymptotic to $e^{2 \phi_{0}} \delta_{\mu \nu}$ rather than $\delta_{\mu \nu}$. This solution is just the "symmetric fivebrane" solution of [15,16] (closely related solutions are discussed in [26,6, 27, 14] ) reinterpreted as a string soliton in six dimensions,

${ }^{4}$ In components $G_{M N}=2 \partial_{[M} A_{N]}, \quad H_{M N P}=3 \partial_{[M} B_{N P]}, \quad F_{M N P Q}^{\prime}=4 \partial_{[M} C_{N P Q]}+$ $8 A_{[M} H_{N P Q]}$. 
and as such much of the following discussion parallels that of [16]. The solution (2.3) is a gravitational analog of a Yang-Mills instanton with the generalized connection given by the sum of the spin connection and the torsion,

$$
\Omega_{+\mu}^{i j}=\omega_{\mu}^{i j}+H_{\mu}^{i j},
$$

being an anti-self-dual $S U(2)$ connection. The solution (2.3) is known to correspond to an exact $(4,4)$ superconformal field theory [15,28] and thus is an exact classical solution of the IIA string theory.

The supersymmetry transformation laws in the soliton background are

$$
\begin{aligned}
\delta \psi_{ \pm M} & =\nabla_{M} \epsilon_{ \pm} \mp \frac{1}{4} H_{M N P} \Gamma^{N P} \epsilon_{ \pm}, \\
\delta \lambda_{\mp} & =\Gamma^{M} \nabla_{M} \phi \epsilon_{ \pm} \pm \frac{1}{6} H_{M N P} \Gamma^{M N P} \epsilon_{ \pm},
\end{aligned}
$$

where $\psi_{ \pm M}$ and $\lambda_{\mp}$ are respectively the gravitinos and dilatinos and the subscript \pm denotes the ten-dimensional chirality. Decomposing the ten-dimensional Lorentz group in accord with $(2.2)$ we have $S O(9,1) \rightarrow S O(4) \times S O(4) \times S O(1,1)$ and

$$
\begin{aligned}
& \epsilon_{+} \rightarrow\left(2_{+}, 2_{+}\right)^{+}+\left(2_{+}, 2_{-}\right)^{-}+\left(2_{-}, 2_{+}\right)^{-}+\left(2_{-}, 2_{-}\right)^{+}, \\
& \epsilon_{-} \rightarrow\left(2_{+}, 2_{+}\right)^{-}+\left(2_{+}, 2_{-}\right)^{+}+\left(2_{-}, 2_{+}\right)^{+}+\left(2_{-}, 2_{-}\right)^{-},
\end{aligned}
$$

where subscripts refer to the the $S O(4)$ chirality and the superscript indicates the $S O(1,1)$ chirality.

The vacuum configuration on $T^{4}$ preserves all of the supersymmetries (2.7) and leads to a six-dimensional theory with $(2,2)$ six-dimensional supersymmetry. The soliton string configuration (2.3) breaks the $\epsilon_{+}$supersymmetries which transform as $2_{+}$under the second $S O(4)$ factor and the $\epsilon_{-}$supersymmetries which transform as $2_{-}$. Thus on $T^{4}$ the completely unbroken supersymmetries are

$$
\left(2_{+}, 2_{-}\right)^{-}+\left(2_{-}, 2_{-}\right)^{+}+\left(2_{+}, 2_{+}\right)^{-}+\left(2_{-}, 2_{+}\right)^{+}
$$

and the supersymmetries broken by the string soliton are

$$
\left(2_{+}, 2_{+}\right)^{+}+\left(2_{-}, 2_{+}\right)^{-}+\left(2_{+}, 2_{-}\right)^{+}+\left(2_{-}, 2_{-}\right)^{-} .
$$

The latter lead to fermion zero modes on the string soliton. 
The vacuum configuration on $K 3$ preserves the supersymmetries $\left(2_{+}, 2_{+}\right)^{+}+\left(2_{+}, 2_{-}\right)^{-}+$ $\left(2_{+}, 2_{+}\right)^{-}+\left(2_{+}, 2_{-}\right)^{+}$and leads to a theory with $(1,1)$ six-dimensional supersymmetry. The string soliton background breaks an additional half of the supersymmetries. The completely unbroken supersymmetries are

$$
\left(2_{+}, 2_{-}\right)^{-}+\left(2_{+}, 2_{+}\right)^{-}
$$

while the supersymmetries unbroken by $K 3$ but broken by the soliton string background are

$$
\left(2_{+}, 2_{+}\right)^{+}+\left(2_{+}, 2_{-}\right)^{+} \text {. }
$$

These broken supersymmetries lead to 8 real supertranslation zero modes on the string 229 which are clearly chiral on the world sheet. We will refer to these as right-moving fermion zero modes. Thus the non-chiral IIA string theory when compactified on $K 3$ leads to a chiral static worldsheet action for the string soliton.

The most obvious bosonic zero modes of the soliton string are the four translation zero modes associated to translation in the four dimensions transverse to the string. Introducing four collective coordinates $X^{\mu}(\sigma)$ for these fields one finds the effective action [

$$
\frac{N}{\alpha^{\prime}} \int d^{2} \sigma e^{-2 \phi}\left(\eta^{\alpha \beta} \partial_{\alpha} X^{\mu} \partial_{\beta} X^{\nu} g_{\mu \nu}+\epsilon^{\alpha \beta} \partial_{\alpha} X^{\mu} \partial_{\beta} X^{\nu} \tilde{B}_{\mu \nu}\right)
$$

where $N$ is a normalization factor (proportional to $V_{K} / \alpha^{\prime 2}$ with $V_{K}$ the volume of $K$ ) and $\tilde{B}$ is the dual Kalb-Ramond field defined by $e^{-2 \phi} * H=d \tilde{B}$ in six dimensions. This is a static gauge $\left(X^{\alpha}=\sigma^{\alpha}\right)$ form of the Polyakov or Nambu action. As argued in [6] (in the context of fivebranes) the powers of $e^{-2 \phi}$ are simply determined by scaling properties of the solutions under constant shifts of $\phi$, and the coupling to $\tilde{B}$ follows from the required violation of the Bianchi identity for $H$ in the presence of the soliton string. The former statement has the immediate interesting consequence, as emphasized in [17], that quantum corrections on the string soliton worldsheet are controlled by the loop expansion parameter of the original string theory.

The unbroken spacetime supersymmetries imply worldsheet supersymmetry [29], which in turn requires the existence of four additional bosonic zero modes to pair with the right-moving fermion zero modes. Worldsheet supersymmetry makes no obvious prediction for left-moving bosonic zero modes. Extra bosonic zero modes arise by considering

5 See references [3],6, 16] for a more complete discussion of this type of effective action. 
the equation for small fluctuations of the three-form potential $C$ in the soliton background. In ten-dimensional form this equation is

$$
d * F^{\prime}=-2 F \wedge H
$$

To solve this we write an ansatz 6

$$
C=\frac{\alpha^{\prime}}{2 \pi} X^{I}(\sigma) U_{I}(y) \wedge d e^{2 \phi_{0}-2 \phi(x)},
$$

with $X^{I}$ a worldsheet zero mode, $e^{2 \phi(x)}$ the background dilaton field of the string soliton and $I=1, \ldots b_{2}$ with $b_{2}$ the second Betti number of $K$. The harmonic two-forms $U_{I}$ comprise an integral basis for $H^{2}(K, Z)$. Note that 2.14) is normalizable and localized near the "throat" of the string soliton. The equation of motion (2.13) then requires that $X^{I}$ be a free two-dimensional field and, using $H=-\hat{*} d \phi$ and $d H=0$, that

$$
d X^{I} \wedge U_{I}=\hat{*} d X^{I} \wedge \hat{*} U_{I}
$$

We use $*$ for the ten-dimensional Hodge dual and wherever $\hat{*}$ appears acting on a form tangent to one component of the decomposition (2.2) it acts as the Hodge dual within that component.

Since the Hodge dual of a harmonic form is harmonic, the duals of the $U_{I}$ may be expressed as a linear combination

$$
\hat{*} U_{I}=U_{J} H_{I}^{J},
$$

where $H^{J}{ }_{I}$ depends on the moduli of $K$. This enables us to rewrite the constraint (2.15) as

$$
\partial_{ \pm} X^{I}= \pm H_{J}^{I} \partial_{ \pm} X^{J}
$$

where $\partial_{ \pm}$are left and right worldsheet derivatives. The fact that $\hat{*} \hat{*}=1 \mathrm{implies}$

$$
H_{J}^{I} H_{K}^{J}=\delta_{K}^{I}
$$

so that $H$ has $b_{2}^{ \pm}$eigenvalues \pm 1 , where $b_{2}^{ \pm}$is the number of self-dual (anti-self-dual) two forms on $K$.

\footnotetext{
6 This corrects equation 5.10 of [16].
} 
An action for the $X^{I}$ can be obtained by dimensional reduction of the ten dimensional action (2.1). After integrating the zero mode wave functions (2.14) over $K \times R^{4}$ one obtains

$$
S_{X^{I}}=\frac{1}{4 \pi} \int d^{2} \sigma L_{I J} \partial_{+} X^{I} \partial_{-} X^{J},
$$

where

$$
L_{I J} \equiv \int_{K} U_{I} \wedge U_{J}
$$

is the intersection matrix on $H^{2}(K, Z)$. This action is of course subject to the chiral constraint (2.17). We further note that $\hat{*} \hat{*}=1$ implies

$$
H^{K}{ }_{I} L_{J K}=L_{I K} H^{K}{ }_{J} .
$$

We are still short one bosonic zero mode needed for worldsheet supersymmetry. The missing zero mode involves a combination of $C$ and the one-form potential $A$ whose field strength $G$ obeys the equation of motion

$$
d * G=-2 * F^{\prime} \wedge H .
$$

A solution of (2.22) and (2.13) is given by

$$
\begin{aligned}
& A=\frac{1}{2 \pi} X^{0}(\sigma) d e^{2 \phi_{0}-2 \phi}, \\
& C=-\frac{1}{\pi} X^{0}(\sigma) e^{2 \phi_{0}-2 \phi} H,
\end{aligned}
$$

provided that $X(\sigma)$ is a free two-dimensional field. This thus gives an additional $(1,1)$ (left,right)-moving bosonic zero mode. Note that since under abelian gauge transformations $\delta A=d \epsilon$ and $\delta C=-2 \epsilon H$, this zero mode is equivalent under a 'large' gauge transformation to a configuration with vanishing $C . X^{0}$ is governed by the action

$$
S_{X^{0}}=\frac{V_{K}}{2 \pi \alpha^{\prime 2}} \int d^{2} \sigma \partial_{+} X^{0} \partial_{-} X^{0} .
$$

Naively the worldsheet fields $X^{I}$ have a noncompact range, and the solitons have a continuous spectrum and pathological thermodynamics. However we believe the $X^{I} \mathrm{~S}$ should be periodically identified for the following reason. In ten dimensions there exists a conserved "electric" charge

$$
q=\int_{\Sigma^{6}} * F
$$


and its "magnetic" dual

$$
\tilde{q}=\int_{\Sigma^{4}} F .
$$

Elementary string states do not carry this charge, but the electric (magnetic) charge is carried by black twobrane (fourbrane) solutions of the theory [30] (as well as by momentum/winding states of fivebranes). If both types of objects are included in the theory, the arguments of [4] then require that the product $q \tilde{q}$ is quantized. We therefore assume a quantization condition

$$
\int_{\Sigma^{4}} F=n g_{4} \alpha^{\prime}, \quad n \in Z,
$$

for some dimensionless minimal magnetic charge $g_{4} . g_{4}$ does not enter in to any perturbative calculation. Later we shall fix $g_{4}$ by nonperturbative considerations.

As in ordinary electromagnetism, if the charges of all states are quantized, gauge transformations are periodic. The theory is invariant under two-form gauge transformations

$$
\delta C=d \epsilon_{2} .
$$

Under such a transformation a state corresponding to a minimal electrically-charged twobrane with a spatial surface $\Sigma_{2}$ acquires a phase

$$
\exp \left(\frac{2 \pi i}{g_{4} \alpha^{\prime}} \int_{\Sigma_{2}} \epsilon_{2}\right) .
$$

If $\epsilon_{2}$ is $g_{4} \alpha^{\prime}$ times an element of $H^{2}(K, Z)$ this will be unity for all twobranes. We should therefore identify

$$
\epsilon_{2} \sim \epsilon_{2}+g_{4} \alpha^{\prime} n^{I} U_{I},
$$

for arbitrary integers $n^{I}$.

For constant $X^{I}$, the zero mode (2.14) is a gauge transformation with parameter

$$
\epsilon_{2}=\frac{\alpha^{\prime}}{2 \pi} X^{I} U_{I}
$$

far from the soliton. The identification (2.30) therefore implies the key identification

$$
X^{I} \sim X^{I}+2 \pi g_{4} n^{I} .
$$

The conditions on the $X^{I}$ s may look a little more familiar in terms of the rotated fields

$$
Y^{J}=O^{J}{ }_{I} X^{I},
$$


where $O$ is an element of $S O\left(b_{2}^{+}, b_{2}^{-}\right)$obeying

$$
O_{K}^{I} H_{L}^{K}\left(O^{-1}\right)_{J}^{L}=\eta_{J}^{I}
$$

and $\eta$ is diagonal with $b_{2}^{+}$plus ones and $b_{2}^{-}$minus ones. The $Y^{I} \mathrm{~s}$ are pure left or pure right-moving fields,

$$
\partial_{ \pm} Y^{I}= \pm \eta_{J}^{I} \partial_{ \pm} Y^{J}
$$

with metric $O L O^{T}$. The identifications (2.32) become

$$
Y^{I} \sim Y^{I}+O^{I}{ }_{J} n^{J} 2 \pi g_{4}
$$

This identification defines a lattice whose vectors are $\mathrm{Ong}_{4}$. The inner product of two lattice vectors is

$$
O_{J}^{I} n^{J}\left[\left(O^{-1}\right)^{M}{ }_{I} L_{M N}\left(O^{-1}\right)^{N}{ }_{K}\right] O^{K}{ }_{L} m^{L} g_{4}^{2}=n^{I} L_{I J} m^{J} g_{4}^{2}
$$

Properties of the intersection matrix $L$ on $H^{2}(K, Z)$ then imply that this is an even selfdual Lorentzian lattice if and only if

$$
g_{4}=1
$$

We henceforth assume this to be the case.

Similar considerations suggest a quantization condition

$$
\int_{\Sigma^{2}} G=n g_{2}, \quad n \in Z
$$

As in the preceding this leads to the periodic identification

$$
X^{0} \sim X^{0}+2 \pi g_{2}
$$

When the Kalb-Ramond field $B$ is a nontrivial element of $H^{2}$ on $K$, the $\int F \wedge F \wedge B$ term in (2.1) leads to a shift in the quantization condition. We have not worked this out in detail, but the modified quantization condition will mix up $X^{0}$ and $X^{I}$, and will lead to dependence of the worldsheet theory on the moduli corresponding to $B$.

When the theory is reduced to six dimensions on $K$ there are $2+b_{2}(K) U(1)$ gauge fields in the low-energy theory which come from Ramond-Ramond fields. One of these comes from the original $U(1)$ field strength $G$. A second arises from the dual field strength $\hat{*} F$ which is a two-form in six dimensions. The other $b_{2}(K)$ gauge fields arise from writing 
$C=U_{I} \wedge A^{I}$ with $A^{I}$ one-form gauge potentials in six dimensions. Since the $X^{I}$ at zero-momentum are pure gauge the periodicity of the $X^{I}$ is equivalent to saying that the six-dimensional $U(1)$ gauge groups are compact.

We can now summarize our results for the two choices $K=K 3, T^{4}$.

\section{K3}

For $K 3, b_{2}^{+}(K 3)=3, b_{2}^{-}(K 3)=19$ and

$$
L_{I J}=\left[\Gamma_{8} \oplus \Gamma_{8} \oplus \sigma^{1} \oplus \sigma^{1} \oplus \sigma^{1}\right]_{I J}, \quad \sigma^{1}=\left(\begin{array}{ll}
0 & 1 \\
1 & 0
\end{array}\right)
$$

where $\Gamma_{8}$ is the Cartan matrix for $E_{8}$. The zero modes (2.14) and (2.23) therefore give $(20,4)$ (left,right)-moving bosonic zero modes with periodicities given by (2.32) and (2.40). It follows from (2.17) that these zero modes live on an even self-dual $(20,4)$ Lorentzian lattice. There are also 8 right-moving worldsheet fermions as required by the unbroken supersymmetries, which transform in the $\left(2_{+}, 2_{+}\right)^{+}+\left(2_{+}, 2_{-}\right)^{+}$of the spacetime $S O(4) \times$ $S O(4) \times S O(1,1)$. Together with the transverse bosonic coordinates of the string, this gives the Green-Schwarz, static gauge worldsheet theory of a Narain compactification to six dimensions of the heterotic string. So, we have finally learned "why" the $K 3$ intersection matrix and the heterotic string both involve $E_{8} \times E_{8}$ !

The moduli space of Ricci flat metrics on $K 3$ is

$$
\mathcal{N}=S O(19,3 ; Z) \backslash S O(19,3 ; R) / S O(19 ; R) \times S O(3 ; R)
$$

As the $K 3$ space is varied, the condition (2.17) varies, and the dual soliton varies over a 58 dimensional subspace of Narain compactifications. Inclusion of the effects of $B$ introduces 22 additional moduli. It is known [20] that these moduli complete the space (2.42) to

$$
\mathcal{M}=S O(20,4 ; Z) \backslash S O(20,4 ; R) / S O(20 ; R) \times S O(4 ; R)
$$

Although we have not worked out the details of the effects of the $B$ moduli on the zero mode quantization, one presumably obtains the full space (2.43) from the collective coordinate expansion of the heterotic soliton string, now viewed as the moduli space of Narain compactifications.

Our collective coordinate expansion provides a precise map from the moduli space of $K 3$ compactifications of the IIA string to $T^{4}$ compactifications of the heterotic string. It 
will be interesting to study the precise structure of this map for various degenerations of the $K 3$ moduli space.

$\mathbf{T}^{4}$

For $T^{4}, b_{2}^{+}=b_{2}^{-}=3$ and

$$
L_{I J}=\left[\sigma^{1} \oplus \sigma^{1} \oplus \sigma^{1}\right]_{I J}
$$

$X^{I}$ and $X^{0}$ therefore parameterize a self-dual $(4,4)$ Lorentzian lattice. This is related to the lattice defining the original $T^{4}$ in a non-trivial but calculable manner. In this case the compactification does not break any supersymmetries, and the supersymmetries which are unbroken by the soliton imply both left and right worldsheet supersymmetry. Invoking the equivalence [31,32] (under $T$-duality) of toroidally compactified IIA and IIB strings, this soliton string may be identified as either a IIA or a IIB string with different target tori.

\section{Eleven-Dimensional Supergravity}

It was conjectured in [13,21] that there should be an exact duality of elevendimensional supergravity, defined as a fundamental supermembrane theory, with the tendimensional type IIA theory. In [9] evidence for such a relation was given at the level of their low-energy effective field theories, where one can avoid the sticky issue of quantization of supermembranes. Together with other observations, this would imply a duality relating $d=11$ supergravity compactified on $K 3$ to the heterotic string compactified on $T^{3}$. Evidence for this is provided by the fact that $d=11$ supergravity compactified on $K 3$ has string solitons whose worldsheet dynamics are precisely those of the heterotic string compactified on $T^{3}$. This follow easily from the results of the previous section. Since the low-energy effective IIA theory was originally obtained [25] by compactification of $d=11$ supergravity, the equations which must be solved are quite similar. The $d=11$ theory contains a fivebrane solution [33,16] which becomes a string when four of its dimensions wrap around the $K 3$. One finds all the zero modes (2.14) in a nearly identical construction. The extra zero mode (2.23) is absent however because the corresponding fields arise only after reduction to $d=10$. Thus one obtains the desired heterotic soliton string with bosonic fields living on a $(19,3)$ Lorentzian lattice. Perhaps, following the lead taken in [21], one may view $d=11$ supergravity on $K 3$ as a consistent quantum theory whose perturbative quantization is defined by a strong-coupling expansion of quantized string solitons rather than as a weak coupling expansion of the original fields! 


\section{Type IIB String Duality}

We now turn to the chiral type IIB theory [34]. In ten dimensions, the IIB theory involves a five-form field strength $F$ which is self-dual. Because of this there is no simple covariant action for the IIB theory. The field content, equations of motion, and on-shell supersymmetry transformation laws are known and are sufficient to discuss the general properties of the solution. The bosonic fields that enter into the fermion transformation laws are (in unitary gauge [34]), in addition to the metric and $F$, a complex closed threeform field strength $H=d B$ and a complex scalar $\mathcal{B}$ obeying $|\mathcal{B}|<1$. The ten-dimensional supersymmetry transformation laws of the fermion fields in the soliton background are given by

$$
\begin{aligned}
\delta \lambda & =i \Gamma^{M} P_{M} \epsilon^{*}-\frac{i}{24} \Gamma^{M N P} G_{M N P} \epsilon \\
\delta \psi_{M} & =D_{M} \epsilon+\frac{i}{480} \Gamma^{P Q R S T} F_{P Q R S T} \Gamma_{M} \epsilon+\frac{1}{96}\left(\Gamma_{M}^{N P Q} G_{N P Q}-9 \Gamma^{N P} G_{M N P}\right) \epsilon^{*},
\end{aligned}
$$

where

$$
\begin{aligned}
P_{M} & =\frac{\partial_{M} \mathcal{B}}{1-\mathcal{B}^{*} \mathcal{B}}, \\
G_{M N P} & =\frac{H_{M N P}-\mathcal{B} H_{M N P}^{*}}{\sqrt{1-\mathcal{B}^{*} \mathcal{B}}},
\end{aligned}
$$

in the notation of 34, 35] (with $\kappa=1$ and $\Gamma_{11} \epsilon=-\epsilon$ ). We consider this theory in the spacetime

$$
T^{4} \times R^{4} \times M^{1,1} .
$$

(Soliton strings with $K 3$ compactification are apparently non-critical strings.) For the string soliton, the non-zero components of the fields are

$$
\begin{aligned}
P_{\mu} & =\frac{1}{2} \nabla_{\mu} \phi, \\
G_{\mu \nu \rho} & =-2 \epsilon_{\mu \nu \rho}{ }^{\lambda} \nabla_{\lambda} \phi, \\
\mathcal{B} & =\tanh \frac{\phi}{2}, \\
e^{2 \phi} & =e^{2 \phi_{0}}+\frac{\alpha^{\prime}}{x^{2}}, \\
g_{\mu \nu} & =e^{3 \phi / 2} \delta_{\mu \nu}, \\
g_{\alpha \beta} & =e^{-\phi / 2} \eta_{\alpha \beta} .
\end{aligned}
$$

The metric which appears in these equations is the "standard" general relativity metric and is related to the string sigma model metric by a factor of $e^{-\phi / 2}$. 
For this field configuration, $\delta \psi$ and $\delta \lambda$ vanish for 16 choices of the spinor $\epsilon$. The other 16 generate 16 fermion zero modes. Worldsheet supersymmetry implies that in static gauge the number of fermi fields must be twice the number of bose fields. There are the usual four bosonic translation zero modes, but we are still short four bosonic zero modes. The four extra zero modes are contained in the excitations of antisymmetric tensor fields with the zero mode field strengths given by

$$
\begin{aligned}
& F_{0}=e^{-\phi / 2} d X_{ \pm}^{I}(\sigma)\left(V_{I} \wedge G \mp 2 W_{I} \wedge d \phi\right), \\
& G_{0}=4 i e^{-\phi / 2} d X_{ \pm}^{I}(\sigma) V_{I} d \phi .
\end{aligned}
$$

where $V_{I}\left(W_{I}\right)$ comprises a harmonic basis for $H^{1}\left(T^{4}, Z\right)\left(H^{3}\left(T^{4}, Z\right)\right)$. For pure right or left-moving excitations the ansatz (4.5) preserves half of the supersymmetry preserved by the solution (4.4) and it is easy to check that $F_{0}$ is a self-dual five-form provided

$$
d X_{ \pm}^{I}= \pm H_{J}^{I} \hat{*} d X_{ \pm}^{J}
$$

where $H_{J}^{I}$ relates the Hodge-dual and Poincare-dual bases

$$
H^{K}{ }_{I} W_{K}=\hat{*} V_{I} .
$$

As before a quantization conditon on the RR charges will lead to periodic identifications of the zero modes. Equation (4.6) then gives four left-moving and four right-moving worldsheet bosons on a $(4,4)$ self-dual Lorentzian lattice. Unbroken worldsheet supersymmetries then relate these to both left and right-moving superpartners, and one obtains a type II worldsheet.

\section{Extended Duality in Type II String Theories}

This is not the end of the story for the IIB string. In fact there are more string solutions in six dimensions. To see this let us first go back to ten dimensions, in which there is a fundamental string solution [2,3] with a singularity at the origin corresponding to an macroscopic fundamental string as a source.

The equations describing the low-energy effective IIB dynamics have an $S U(1,1)$ symmetry. As noted in [16], charge quantization clearly forbids a continuous symmetry, but allows a discrete $S L(2, Z)$ subgroup which was conjectured in [13] to be an exact symmetry

7 This corrects formula 5.6 of [16. 
of the ten-dimensional theory. Under this $S L(2, Z)$ the real and imaginary parts $H_{1}$ and $\mathrm{H}_{2}$ of $\mathrm{H}$ are a doublet

$$
\left(H_{1}, H_{2}\right) \rightarrow\left(a H_{1}+b H_{2}, c H_{1}+d H_{2}\right)
$$

with $a, b, c, d \in Z$ and $a d-b c=1$. Since everything transforms covariantly under these transformations one obtains new string solitons.

The usual fundamental string of [2, 3] acts as a source for the usual NS-NS KalbRamond field $H_{1}$. Under the $S L(2, Z)$ transformation $H_{1} \rightarrow H_{2}$ this transforms into a string which acts as a source for the R-R Kalb-Ramond field $H_{2}$. This dual string is a natural candidate for a fundamental string of a strongly coupled phase of the IIB theory. Upon toroidal reduction to six dimensions, $S L(2, Z)$ combines with the $T$-duality group to give an $S L(5, Z)$ symmetry of the equations of motion. This leads to a large multiplet of string solutions, which includes both the fundamental and solitonic type solutions.

\section{Discussion and Conclusions}

In order to put our results in proper perspective, it is useful to draw an analogy with $d=4$ super Yang-Mills theory. For the case of $N=4$ supersymmetries, the low-energy effective field theory is (at generic points in the moduli space) an abelian theory, and has an $S$-duality symmetry which exchanges weak and strong coupling. It has been conjectured [36] that this extends to an exact symmetry of the theory. If true, the conjecture implies that there are two microscopic definitions (which involve different coupling constants) of every physical theory. The symmetry would then constrain not just the leading term in the low-energy effective action, but every term in a derivative expansion of the exact theory. A key observation [37,38,39] which makes this conjecture plausible is that the symmetry does extend beyond the low-energy effective action at least to the spectrum of stable BPS saturated states.

It is also true, as has recently understood in detail [40], that for pure $N=2$ Yang-Mills theory the low-energy effective field theory is (at generic points in the moduli space) an abelian theory, and has an $S$-duality symmetry which exchanges weak and strong coupling. However it is not plausible, and has not been proposed, that this extends to exact symmetry of the theory (although the effective symmetry is nevertheless extremely useful). Indeed the symmetry can not even be extended to the BPS states because there are no spin one 
monopoles. Thus the $N=2$ theory has an effective $S$-duality, in contrast to the $N=4$ theory which may have an exact $S$-duality.

In many regards, discussions of string-string duality to date are somewhat like discussing $S$-duality in $d=4$ Yang-Mills theory without knowing the spectrum of BPS monopoles. Most of the discussions involve only the low-energy effective field theory and do not really distinguish between exact and effective duality 8 . The duals of the string winding and momentum states on $T^{4}$, and in particular the exponential spectrum of such states which are BPS saturated, involve charged RR states in the IIA theory and little is known about the properties of such states. One can argue that such states must exist since one can construct black holes carrying RR charge, but detailed questions involving counting of states have not been successfully addressed from this point of view. We will argue that there is a much more concrete picture of these states which follows from our construction, which therefore provides strong evidence for an exact duality.

The heterotic soliton of the Type IIA theory on K3 carries currents which couple to the $U(1)^{24}$ low-energy gauge fields coming from the Ramond-Ramond (RR) sector of the IIA theory. If we consider instead of an infinite soliton string a finite closed loop of string, then at first we might expect that it can shrink to zero size and disappear since it is topologically trivial 9 . However this is not the case if it carries non-zero RR charge. For example, a closed loop of string where the coordinates $X^{I}$ come back to themselves up to a shift by an element of the lattice defined by (2.36) when going around the string carries such charge. Since there are no such charged states in the perturbative spectrum and since RR charge is conserved, such a string configuration cannot disappear. It presumably shrinks down to a tiny loop which is a BPS saturated charged RR state.

Furthermore, when the IIA theory is strongly coupled the soliton string becomes weakly coupled and has a string tension which is much smaller than the fundamental string tension. It seems likely then that at strong coupling in the IIA theory the low-lying spectrum can be obtained by quantizing the soliton string as a weakly coupled fundamental string. This clearly leads to the detailed spectrum of BPS saturated, charged RR states which are required by string-string-duality and also to enhanced gauge symmetries at the

8 An exception to this, emphasized in [9] and implicit in [13], is the observation that a consistent implementation of the duality of dualities requires properties of massive string states which go beyond low-energy supergravity.

9 Although this is not always the case. See for example the discussion in 41. 
special points in the $K 3$ moduli space which map to enhanced symmetry points in the Narain moduli space. One obvious question that arises in this point of view is whether this will "double count" neutral states such as the graviton, once as excitations of the fundamental string and once as excitations of the soliton string. We believe the correct point of view is that these are two dual descriptions of the same state, analogous to the description of the photon in $N=4$ Yang-Mills theory as either an "electric" neutral gauge boson at strong coupling or a "magnetic" neutral gauge boson at weak coupling.

In some sense the case for an exact six-dimensional string-string duality is even more compelling than that for an exact $S$-duality in $N=4, D=4$ Yang-Mills. In the latter case the spectrum of BPS states is largely fixed by supersymmetry, so there was little room for disagreement with the predictions of $S$-duality. In the string case supersymmetry allows infinitely many possibilities for the spectrum of the solitonic string, so the emergence of the heterotic string is quite striking.

The construction dual to the one presented here would be the construction of a IIA string on $K 3$ as a soliton in the heterotic string on $T^{4}$. A natural candidate exists for such a solution, it is again the symmetric fivebrane, now interpreted as a solution with non-zero gauge fields describing a Yang-Mills instanton. However at a generic point in the moduli space of heterotic strings on $T^{4}$ the gauge symmetry is $U(1)^{24}$ and there are no finite size instanton solutions, at least in the field theory limit. At points with enhanced gauge symmetry there are finite size instantons but there is no obvious sign of the $K 3$ factor in the instanton moduli space which would be needed to give the correct IIA soliton string. This is just a higher-dimensional version of the conundrum which arises in the study of $H$-monopoles and $S$-duality in four dimensions where again a $K 3$ factor in the instanton moduli space seems to be required for the correct counting of states [23]. This is perhaps not surprising given the fact that six-dimensional string-string duality can be related to four-dimensional $S$-duality. A resolution of this puzzle is likely to play an important role in a deeper understanding of duality in string theory.

In closing we mention that the six-dimensional theories have additional $p$-brane solitons corresponding to fivebranes which wrap around cycles of $K 3$ or $T^{4}$ with dimension less than four. It is of interest to understand how these solitons behave under various duality transformations, and in particular which of them become light at strong coupling. Clearly at present that duality can be best understood in those contexts that involve just strings and not other $p$-brane like solutions. On the other hand the close relation between six-dimensional string solutions and $p$-brane solutions in higher dimensions suggests that 
we have not yet heard the end of the story as far as $p$-branes are concerned. The precise interplay between the treatment of the theory in ten and six dimensions and the role, if any, played by $p$-branes other than strings should be fascinating.

Note Added: After completion of this work, an interesting paper appeared [42] in which the external field configuration to which charged excitations of the soliton described herein give rise was constructed by dualizing the results of [24].

\section{Acknowledgements}

We thank J. Gauntlett, E. Martinec and G. Moore for discussion. The results on sixdimensional string-string duality in toroidal type II compactifications were mostly obtained

several years ago in the context of ongoing collaboration with C. Callan, and we thank him for collaboration and discussions at that time. This work was supported in part by NSF Grant No. PHY 91-23780 and DOE Grant No. DOE-91ER40618. 


\section{References}

[1] H. Nielsen and P. Olesen, Nucl. Phys. B61 (1973) 45.

[2] A. Dabholkar and J. A. Harvey, "Nonrenormalization of the Superstring Tension," Phys. Rev. Lett. 63 (1989) 478.

[3] A. Dabholkar, G. Gibbons, J. A. Harvey and F. R. Ruiz, "Superstrings and Solitons, " Nucl. Phys. B340 (1990) 33.

[4] R. Nepomechie, Phys. Rev. D31 (1985) 1921; C. Teitelboim, Phys. Lett. B176 (1986) 69.

[5] M. J Duff, "Supermembranes, the First Fifteen Weeks, " Class. Quant. Grav. 5 (1988) 189.

[6] A. Strominger, "Heterotic Solitons, " Nucl. Phys. B343 (1990) 167; E: Nucl. Phys. B353 (1991) 565.

[7] P.K. Townsend, "Three Lectures on Supermembranes" in Superstrings '88 eds. M. Green, M. Grisaru, R. Iengo, E. Sezgin and A. Strominger, World Scientific, Singapore, 1989.

[8] M. J. Duff, "Strong/Weak Coupling Duality from the Dual String," NI-94-033, CTPTAMU-49/94, hep-th/9501030.

[9] E. Witten, "String Theory Dynamics in Various Dimensions," hep-th/9503124.

[10] J. Schwarz and A. Sen, Phys. Lett. B312 (1993) 105, hepth/9305185; P. Binetruy, Phys. Lett. B315 (1993) 80, hep-th/9305069.

[11] A. Font, L. Ibanez, D. Lust, and F. Quevedo, Phys. Lett. 249B (1990) 35; S. J. Rey, Phys. Rev. D43 (1991) 526; (1992) 374; J. Harvey, J. Gauntlett and J. Liu, Nucl. Phys. 409B (1993) 363; R. Khuri, Phys. Lett. 259B (1991) 261; J.H. Schwarz and A. Sen, Nucl. Phys. 404B (1993) 109; J. Gauntlett and J. Harvey, hep-th/9407111; A. Sen, Int. J. Mod. Phys. A9 (1994) 3707, hep-th/9402002; J. H. Schwarz, hep-th/9411178, C. Vafa and E. Witten, hep-th/9408074; Nucl. Phys. B431 (1994) 3, L. Girardello, A. Giveon, M. Porrati and A. Zaffaroni, Phys.Lett. B334 (1994) 331, hep-th/9406128; J. Harvey, G. Moore and A. Strominger, hep-th/9501022; M. Bershadsky, A. Johansen, V. Sadov and C. Vafa, hep-th/9501096.

[12] For a review see M. J. Duff, R. R. Khuri, and J. X. Lu, "String Solitons," hepth/9412184.

[13] C.M. Hull and P.K. Townsend, "Unity of Superstring Dualites," QMW-94-30, $\mathrm{R} / 94 / 33$, hep-th/9410167.

[14] M. J. Duff and J. X. Lu, "Elementary Fivebrane Solutions of D=10 Supergravity," Nucl. Phys. B354 (1991) 141.

[15] C.G. Callan, J. A. Harvey and A. Strominger, "Worldsheet Approach to Heterotic Instantons and Solitons," Nucl. Phys. 359 (1991) 611. 
[16] C. G. Callan, J. A. Harvey and A. Strominger, "Worldbrane Actions for String Solitons," Nucl. Phys. B367 (1991) 60.

[17] M. J. Duff and J. X. Lu, "Remarks on String/Fivebrane Duality," Nucl. Phys. B354 (1991) 129; "String/Fivebrane Duality, Loop Expansions and the Cosmological Constant," Nucl. Phys. B357 (1991) 534.

[18] A. Ceresole, R. D'Auria, S. Ferrara and A. Van Proeyen, "Duality Transformations in Supersymmetric Yang-Mills Theory Coupled to Supergravity, "hep-th/9502072.

[19] C. Vafa, as referenced in [9].

[20] N. Seiberg, "Observations on the Moduli Space of Superconformal Field Theories, "Nucl. Phys. B303, (1986), 288; P. Aspinwall and D. Morrison, "String Theory on K3 Surfaces," preprint DUK-TH-94-68, IASSNS-HEP-94/23, hep-th/9404151.

[21] P.K. Townsend, "The Eleven-Dimensional Supermembrane Revisited," R/95/2, hepth/9501068.

[22] J. Polchinski and A. Strominger, "Effective String Theory," Phys. Rev. Lett. 67 (1991) 1681.

[23] J. Gauntlett and J. A. Harvey, "S-duality and the Spectrum of Magnetic Monopoles in Heterotic String Theory, "hep-th/9407111.

[24] A. Sen, "Macroscopic Charged Heterotic String", hep-th/9206016, Nucl. Phys. B388 (1992) 457.

[25] I. C. G. Campbell and P. West, Nucl. Phys. B243, (1984) 112; F. Giani and M. Pernici, Phys. Rev. D30, (1984) 325; M. Huq and M. A. Namazie, Class. Quant. Grav. 2 (1985) 293.

[26] A. Strominger, "Superstrings with Torsion", Nucl. Phys. B274 (1986) 253.

[27] S. J. Rey, Phys. Rev. D43 (1991) 526.

[28] For a review see C. G. Callan, J. A. Harvey and A. Strominger, "Supersymmetric String Solitons" in String Theory and Quantum Gravity 1991: Proceedings of the Trieste Spring School, World Scientific, Singapore, 1991, hep-th/9112030.

[29] J. Hughes and J. Polchinski, Nucl. Phys. B278, 147 (1986).

[30] G. Horowitz and A. Strominger, "Black Strings and p-branes," Nucl. Phys. B360 (1991) 197.

[31] J. Dai, R. G. Leigh, and J. Polchinski, "New Connections Between String Theories," Mod. Phys. Lett. A4 (1989) 2073.

[32] M. Dine, P. Huet, and N. Seiberg, "Large and Small Radius in String Theory," Nucl. Phys. B322 (1989) 2073.

[33] R. Güven, "Black $p$-brane solutions of $D=11$ supergravity theory," Phys. Lett. B276 (1992) 49.

[34] J. H. Schwarz, Nucl. Phys. B226 (1983) 269.

[35] M. B. Green, J. H. Schwarz, and E. Witten, Superstring Theory, Vol. 2. , Cambridge University Press (1987). 
[36] C. Montonen and D. Olive, Phys. Lett. 72B (1977) 117.

[37] H. Osborne, "Topological Charges for $\mathrm{N}=4$ Supersymmetric Gauge Theories and Monopoles of Spin 1," Phys. Lett. 83B(1979)321.

[38] E. Witten and D. Olive, Phys. Lett. 78B (1978) 97.

[39] A. Sen, Phys. Lett. B329 (1994) 217, hep-th/9402032.

[40] N. Seiberg and E. Witten, "Electromagnetic Duality, Monopole Condensation and Confinement in N=2 Supersymmetric Yang-Mills Theory, "hep-th/9407087.

[41] Y. S. Wu and A. Zee, " A Closed String (or Ring) Soliton Configuration with Nonzero Hopf Number, " Nucl. Phys. B324 (1989) 623.

[42] A. Sen, "String-String Duality Conjecture in Six Dimensions and Charged Solitonic Strings", hep-th/9504027. 